\title{
DIRECT PHASE-DOMAIN CALCULATION OF TRANSMISSION LINE TRANSIENTS USING TWO-SIDED RECURSIONS
}

\author{
George Angelidis \\ Adam Semlyen \\ Department of Electrical and Computer Engineering \\ University of Toronto \\ Toronto, Ontario. Canada M5S 1A4
}

\begin{abstract}
This paper presents a new method for the simulation of electromagnetic transients on transmission lines. Instead of using convolutions of the input variables only, we perform short convolutions with both input and output variables. The result is a method of Two-Sided Recursions (TSR), which is comparable in efficiency with the existing recursive convolutions or with their equivalent state variable formulations. It is, however, conceptually simpler and can be applied, in addition to fast modal-domain solutions, to the direct phase-domain calculation of transmission line transients with very accurate results.
\end{abstract}

Keywords: Transmission line transients, Phase-domain. Recursions, Convolutions.

\section{LNTRODUCTION}

This paper describes a new, highly efficient and accurate. methodology for the calculation of electromagnetic transients on transmission lines with frequency dependent parameters. The particular advantage of the method is its applicability to the direct phase-domain computation of the transients. It is based on TwoSided Recursions (TSR), or short convolutions, as opposed to conventional convolutions which is the basis of probably all computational methods used at present.

It is well known that the basic data for computation of transients, the transmission line parameters, are initially defined in the frequency-domain and are frequency dependent. Therefore, basic input/output relations appear at first in the frequency-domain in the form:

$$
y=H u
$$

Their lime-domain equivalent could be written using a convolution with $u(t)$ as input. The notation here and in the following corresponds to vectors and matrices in the general case of phasedomain calculations. but could represent scalars in the case of individual modes. Because $H(s)$ is a transcendental matrix or scalar function of $s$, such convolutions are "long". A first significant simplification was obtained when a recursive scheme was devised [1] for the fast evaluation of such convolutions. Recursive convolutions are equivalent (see the appendix of [1]) to a state variable approach. They require. however. an approximation of the unit step response of $H(s)$ by a small number of exponential functions. In the frequencydomain, this is equivalent to a rational approximation of $H(s)$, whereby (1) becomes:

94 SM 465-5 PWRD A paper recommended and approved by the IEEE Transmission and Distribution Committee of the IEEE Power Engineering Society for presentation at the IEEE/PES 1994 Summer Meeting, San Francisco, CA, July 24 - 28, 1994. Manuscript submitted November 18, 1993; made available for printing May 6, 1994.

$$
y=D^{-1} N u
$$

Here, $D(s)$ and $N(s)$ are polynomial matrix or scalar functions of $s=j \omega$ [2]. High accuracy of this procedure was achieved subsequentiy, as reported in [3]. All computations were performed using modes because rational fitting is very difficult for the matrix problem (1), i.e., for direct phase-domain calculations. However, phase-to-mode transformations are themselves frequency dependent and using a single, real, transformation matrix for time-domain calculations is just an approximation. albeit fully satisfactory for overhead lines [4]. In the case of cables the problem is, however, much more difficult [5].

The new method overcomes the above difficulties. Its main idea was inspired by [6] for scalar conditions. However, (1) and (2), to which it is related. may also be viewed as vector-matrix relations, and (2) can take the following form:

$$
D y=N u
$$

In its time-domain interpretation. (3) suggests two convolutions, rather than just one as for (1), but these are now short for the following reason: both $D(s)$ and $N(s)$ are polynomials in $s$ and the equivalent of $s$ in the time-domain is a derivative or a finite difference. In other words, each power of $s$ requires one past value for the corresponding variable. If the polynomial is of order $n$, then $n$ past values are required. Thus, low order polynomials lead to short convolutions, i.e.. efficient computations. By this simple line of thoughts, we have ended up with the approach of difference equations or Two-Sided Recursions (TSR) and the corresponding formula

$$
\sum_{k=0}^{n} A_{k} y_{r-k}=\sum_{k=0}^{n} B_{k} u_{r-k}
$$

where $r$ indicates the current or present time as it progresses, and $k$ is a backward count for a small number $n$ of past values. Equation (4) can be written as a current-output to current-input relation as follows:

$$
y_{r}=G u_{r}+h_{r}
$$

where $G\left(=A_{o}^{-1} B_{o}\right)$ is constant and $h_{r}$ is a variable "history" term composed of past, known, values of input and output.

Since (4) implies time samples, it could be derived from state equations integrated according to some rule, e.g., trapezoidal. to which it is equivalent. However, we do not need state equations to write the main relation (4): it has been obtained without any mathematical derivation and is handled as a postulated, basic relationship. All that we need for its practical use, either for scalar (i.e., modal) or for matrix (i.e., phase-domain) calculations, is the identification of the TSR coefficients $A_{k}$ and $B_{k}$. This can be done either directly in the time-domain [7] or in the frequency-domain [8], as described in the following. Especially in the matrix case. the direct problem formulation (4) is of great practical importance since the alternative of rational approximation of matrix transfer functions is a much more difficult task [4].

The dynamic input/output relation (4) can be taken as sparse (i.e., some of the TSR coefficients can be assumed to be zero) thus increasing the dynamic order without additional computational burden.

0885-8977/95/\$04.00 (C) 1994 IEEE 
Equation (4) suggests that it has a $z$-domain equivalent to which it is related via a $z$-transform. Reference [9] covers this topic in great detail. We note. however, that our approach is only conceptually related to the existence of a $z$-domain representation, which we never calculate. since (4) is in our approach the equation from where the entire process starts.

The primary application of the new method is the simulation of transients directly in the phase-domain. Here, accuracy is our ultimate goal so that the obtained simulations may even serve as a basis of comparison for results obtained using modes. The procedure may also be used for the calculation of transients in the modaldomain. Then, our goal is speed and efficiency, as ultimate accuracy cannot be obtained due to the limitations inherent in modal transformations

\section{METHODOLOGY}

In this section we first present the general methodology, which basically reflects the approach implemented in the EMTP (ElectroMagnetic Transients Program). for the calculation of transients in transmission lines. It serves to indicate at which place the Two-Sided Recursions (TSR) should the used. Then we discuss the problem of the identification of the TSR coefficients needed for the implementation of the method. Finally, we show how the results of the identification are validated in order to assure accurate transient simulations.

\subsection{Simulation of Transmission Line Transients}

\section{Phase-Domain Equations}

The nodal voltage and the current flowing into a transmission line are given by the frequency-domain (vector) relations

$$
\begin{gathered}
v=v^{\prime}+v^{\prime \prime} \\
i=i^{\prime}+i^{\prime \prime}
\end{gathered}
$$

where prime and double-prime quantities denote incident and reflected waves, respectively. The current-voltage relations are given by

$$
\begin{aligned}
& i^{\prime}=H_{Y} v^{\prime} \\
& i^{\prime \prime}=-H_{Y} v^{\prime \prime}
\end{aligned}
$$

where $H_{Y}$ is the characteristic admittance transfer matrix. defined in Appendix $\mathrm{A}$. The port-to-port relations are given by

$$
\begin{aligned}
& v^{\prime}=H_{P_{v}} v_{f}^{\prime \prime} \\
& i^{\prime}=-H_{P_{i}} i_{f}^{\prime \prime}
\end{aligned}
$$

where $H_{P_{v}}$ and $H_{P_{i}}$ are the voltage and current wave propagation transfer matrices, defined in Appendix $\mathrm{A}$, and the subscript $f$ denotes the far end of the line. Eliminating the reflected wave variables from (6) - (9), yields:

$$
2 i^{\prime}-i=H_{Y} v
$$

Equations (11) and (12) are in the input/output form of (1). Their time-domain equivalents, are the back-bone of traditional transmission line transient simulations $[1-4]$. In this work, the time-domain equivalent of (1) is (4), where the TSR coefficients $A_{k}$ and $B_{k}$ are suitably identified by a procedure outlined in the next subsection. It is then applied to the (matrix-vector) multiplications in (11) and (12).

In the case of the propagation relation (11), because of the travel delay $\tau$, all inputs into (4) are past values and, therefore, in (5) $G=0$. Thus $i^{\prime}$ in (12) is fully known. Consequently, (12) yields the following current-voltage relation for each end of the transmission line:

$$
i_{N}-Y_{N} v=i
$$

where $i_{N}$ and $Y_{N}$ are known quantities.

\section{Norton Equivalents}

Equation (13) allows the representation of the transmission line, at each end, by a Norton equivalent, where $i_{N}$ is the Norton current and $Y_{N}$ is the Norton-source admittance. Similarly, any passive load, machine or other power system component connected to a node. can be suitably represented by a Norton equivalent as (13). Following the standard EMTP procedure, applying Kirchhoff's current law to the sum of equations (13) for all Norton equivalents connected to a node, yields

$$
\sum_{k} i_{N k}-\left(\sum_{k} Y_{N k}\right) v=\sum_{k} i_{k}=0
$$

from where the nodal voltage is obtained. Subsequently, (13) yields the current flowing into each component connected to the node. For transmission lines, the reflected current at each end is then obtained from (7). This concludes the series of transient-simulation calculations at each time step.

\section{Modal-Domain Equations}

Equations (6)-(12) can also be written in the modal-domain. using modal voltages, currents and transformation matrices, as defined in Appendix A. Since the modal transfer functions are scalar, the twosided recursions of (11) and (12) can be performed individually for each mode. Although these calculations can be done in the modaldomain. switching operations always take place in the phase-domain. This dictates the interface of the transmission line with other lines or power system components, i.e., equation (13), to be in the phasedomain. Consequently, a voltage and current transformation from the phase-domain to the modal-domain. and vice-versa, is required at every step of the simulation, using the modal transformation matrices defined in Appendix A. In reality, these matrices are complex and frequency-dependent. However, their variation with frequency is not significant. especially in the case of single-circuit overhead transmission lines. Therefore, for the purpose of overhead transmission line transient simulations, these matrices can be approximated by the real part of the corresponding matrices calculated for a specific frequency. The latter is suitably selected depending on the particular transient study.

\subsection{Identification of TSR Coefficients}

The most direct approach for the identification of the TSR coefficients in (4) is, of course, using (4) itself in the time-domain. with a sufficiently large number of given values for the inputs $u_{r}$ and outputs $y_{r}$. These can be generated by (a) starting with a periodical signal $u(t)$, (b) obtaining by fast Fourier transform (FFT) its frequency spectrum $u(\omega)$, then (c) calculating $y(\omega)$ from (1) where $H(\omega)$ is given in the first place, and, finally, (d) obtaining via inverse FFT the output $y(t)$. We note that (4) is a set of linear homogeneous equations in the elements of $A_{k}$ and $B_{k}$. Therefore, some of these elements must be normalized, i.e., set to some constant values, to obtain a standard least-squares problem. Details about its solution are given, for instance, in [7].

A second altemative for the identification of the TSR coefficients is using frequency-domain fitting. This has the following advantages:

- The initial data for the problem is the given transfer function in the frequency-domain. Therefore, the Fourier transformations, discussed above, are not needed.

- The fitting can be done over a very wide range of frequencies. While an FFT operates over linearly-spaced samples, the direct frequency-domain fitting can use (for example) logarithmically-spaced data and thus cover with few samples a very wide frequency range.

Our experience has confirmed the above advantages of frequencydomain fitting. Therefore, we give below more details about this procedure. 


\section{Frequency-Domain Fitting}

The TSR coefficients of (4) are identified by the least-squares solution of an over-determined system of weighted linear equations. obtained by writting (1) for different frequencies, referred to as fitting frequencies. First we note that (4) is in the discrete time-domain. If the input is of a given frequency $\omega$, then it must have a multiplier $e^{\mathrm{j} \omega}$ and samples of $t$ are to be taken at intervals $\Delta t$. Moreover, since the input is a vector quantity, we have to choose vectors spanning the entire corresponding linear space: a good choice for this is to take the input vectors as the standard basis, i.e., the columns of the identity matrix $I$. Thus the chosen inputs are the columns of $l e^{j \omega t}$ Correspondingly, the outputs, according to (1), will be the columns of $H(j \omega) e^{j \omega t}$. With these sets of input and output vectors, (4) yields:

$$
\sum_{k=0}^{n} A_{k} H(\mathrm{j} \omega) e^{(r-k) j \omega \Delta t}=\sum_{k=0}^{n} B_{k} e^{(r-k) j \omega \Delta t}
$$

Simplifying and rearranging (15), yields

$$
\sum_{k=0}^{n}\left(A_{k} H(\mathrm{j} \omega)-B_{k}\right) e^{-k j \omega \Delta t}=0
$$

where the right-hand-side is a matrix of zeros. Multiplying (16) with suitable weighting factors and decomposing it in real and imaginary parts, the set of these equations for all fitting frequencies yields

$$
C X=0
$$

where $C$ is a matrix of known quantities, and $X$ is a matrix composed of the TSR coefficients, as follows:

$$
X^{\mathrm{T}}=\left[A_{0} A_{1} \cdots A_{n} B_{0} B_{1} \cdots B_{n}\right]
$$

Because of a normalization, not all entries of $X$ are unknown, and can be moved to the right-hand-side in (17). The normalization used was to set $A_{0}$ equal to the identity matrix. Equation (17) is an overdetermined system that is solved for $X$ using a least-squares approach.

\section{Back-Winding}

As seen in relation with the two-sided recursion of (11), the propagation time delay is taken into account by past-shifting the input. Therefore, in the identification of the TSR coefficients for the propagation transfer matrix, we use the "back-wound" transfer matrix, defined as follows:

$$
\hat{H}_{P}(j \omega) \triangleq H_{P}(j \omega) e^{j \omega \tau}
$$

We refer to this operation as back-winding because it removes the spirals of $H_{P}(j \omega)$ on the complex plane [1].

\section{Sparsity}

The method allows for "sparse" TSR coefficients. That is, some of the coefficient matrices in (4) may be zero. The algorithmic implications are minimal, but the advantage is that a high-order model may have only a few coefficients.

\section{Stability}

The stability of equation (4) must be examined. The identification process does not necessarily lead to a stable model. The model is stable when, in the absence of input, i.e., $u(t)=0$, the output $y(t)$ remains bounded. Thus, from (4), for any set of initial values $\left\{y_{0}, y_{1}, \ldots, y_{n-1}\right\}$ the recursion

$$
\sum_{k=0}^{n} A_{k} y_{r-k}=0
$$

must give a stable solution $y_{r}$ for $r \geq n$. This problem, at least for the scalar case, is discussed in the literature in relation with the stability of difference-equations [10]. The coefficients are stable when the magnitude of all the roots of the characteristic matrix polynomial

$$
P_{A}(\lambda) \equiv A_{0} \lambda^{n}+A_{1} \lambda^{n-1}+A_{2} \lambda^{n-2}+\cdots+A_{n}
$$

is less than unity. The roots are the eigenvalues of the corresponding companion matrix, as shown in Appendix B.

We note that in the above stability analysis we have assumed $u(t)$ to be the input. This resulted in condition (21), involving the TSR coefficients $A_{k}$. However, (4) is a symmetrical relation and $y(t)$ could have been chosen as input. Then a relation analogous to (21) would have been obtained for $B_{k}$. In some cases, such as that of the propagation matrix $H_{P}$ in (10) or (11), there is no doubt which variable is the true input, so that no ambiguity can result. However, in the case of $H_{Y}$ in (12) either $v$ or $i$ can be the input (termination in a voltage or current source, respectively) or, more generally, a relation between $v$ and $i$ is imposed by the connected load. In that case, equation (21) should be replaced by a more complex polynomial with coefficients in terms of both $A_{k}$ and $B_{k}$. Details are given in Appendix C.

The stability of the TSR coefficients is affected by the selection of the fitting frequencies as well as the weighting factors at each frequency. The inclusion of a steady-state constraint in the leastsquares solution of (17), significantly facilitates the calculation of stable coefficients. The constraint we use is equation (16) employed for identification, but for zero frequency, i.e.,

$$
\sum_{k=0}^{n}\left(A_{k} H(0)-B_{k}\right)=0
$$

which is equivalent to enforcing zero frequency in (17) by an infinite weighting factor.

\section{Parameters of Identification}

The identification of TSR coefficients is a delicate design procedure that depends on the following input parameters:

1) The time step, $\Delta t$. This is usually a trade-off affected by the highest observable frequency and the time horizon of the transient simulation.

2) The order of the model, $n$. A higher order model is more accurate, but it results in more TSR coefficient matrices, thereby increasing the computational effort during the transient simulation.

3) The fitting frequencies. These span the frequency range where the model is intended to be accurate. For a narrow frequency range, a low-order model is most of the times satisfactory whereas a wide range requires a higher-order model.

4) The weighting factors. These are useful for the fine tuning of the model, for enforcing high accuracy at specific frequencies, e.g., the power frequency, and for achieving stability.

5) The sparsity of the model. In some cases, for the same number of coefficients, a sparse model is more stable.

\section{Modal Identification}

Modal identification is a simpler case since each mode is treated separately and the TSR coefficients are scalars. Individual modal identification allows for very flexible models where the parameters of the identification process can be different for each mode.

\subsection{Validation of TSR Coefficients}

\section{Frequency-Domain Validation}

The frequency-domain validation of the TSR coefficients is very simple. From equation (16) used for identification, the approximated transfer matrix is given by

$$
H(\mathrm{j} \omega)=\left(\sum_{k=0}^{n} A_{k} e^{-k \mathrm{j} \omega \Delta t}\right)^{-1} \sum_{k=0}^{n} B_{k} e^{-k \mathrm{j} \omega \Delta t}
$$

The accuracy of the model is then assessed by comparing the magnitude and phase plots obtained by (23) with the ones obtained from equations (A.4) and (A.6) of Appendix A. 


\section{Time-Domain Validation}

The time-domain validation of the TSR coefficients is not as straightforward. A periodic signal is used as input, and the response of the model is compared to the response calculated using FFT pairs:

$$
y(t)=F^{-1}\{H(s) F\{u(t)\}\}
$$

The input signal chosen was a square-wave between -1 and 1 , so that the output looks somewhat like the unit-step response, but it is periodic. The length of the period depends on the lowest observable frequency. The transition between the values -1 and 1 is smoothed (it takes a few time steps) since the model is not validated for very high frequencies near the theoretical limit of $1 / 2 \Delta$.

\section{RESULTS}

All computations were performed on a $486-\mathrm{DX} / 33 \mathrm{MHz}$ Personal Computer using the MATLAB version 4.0 data analysis and visualization software. The TSR coefficient identification and validation algorithms. and the transient simulation programs were implemented as MATLAB M-script-files. A $300 \mathrm{~km}$ long 3-phase transmission line with horizontal configuration, and a time step of $5 \mu \mathrm{s}$ were used in all simulations.

\subsection{TSR Coefficient Identification and Validation}

\section{Phase-Domain}

For models in the phase-domain, our ultimate goal was accuracy. For overall errors below $2 \%$, a 7 th order model was necessary for the propagation transfer matrix and a 5 th order model for the characteristic admittance transfer matrix.

Figure 1 shows the frequency response of the model. The corner element $H_{P_{i}}(1.1)$ of the current-wave propagation transfer matrix is displayed. Curve 1 is the response obtained from equation (A.4) of Appendix A and Curve 2 is the TSR approximation from equation (23). The model is very accurate in the intended range of $50 \mathrm{~Hz}$ to $50 \mathrm{kHz}$.

Figure 2 shows the time-domain response of the model to a square-wave input applied to phase $a$. The output of phase $a$ is displayed. Curve 1 is calculated by FFT pairs and Curve 2 is the TSR approximation. The two curves are almost identical.

Sparsity did not prove to be particularly useful in the phasedomain model. Dense models were more accurate for the same number of coefficients. Sparsity was only useful for improving the
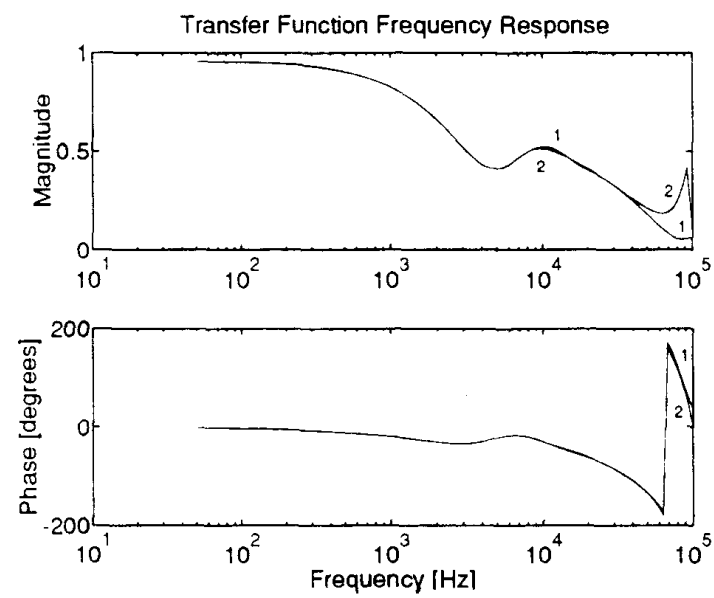

Figure 1. Frequency response of phase-domain propagation model; element $(1,1)$ of matrix $H_{p_{i}}$.

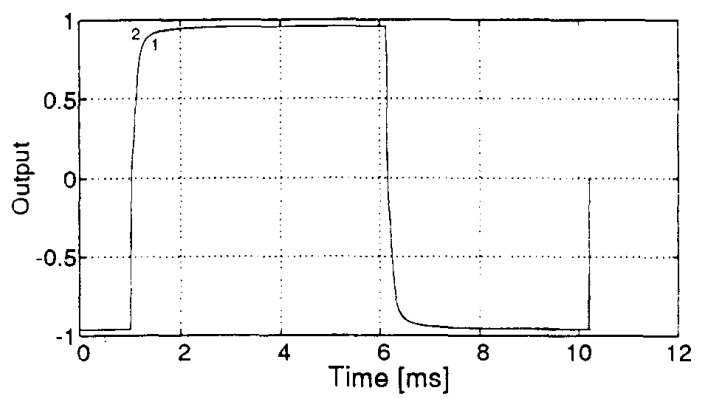

Figure 2. Response to square-wave input of phase-domain propagation model; element (1,I) of matrix $H_{P_{1}}$.

stability of high order models for the characteristic admittance transfer matrix, prompted by the presence of parasitic oscillations in large numbers of dense coefficients.

\section{Modal-Domain}

For models in the modal-domain, our ultimate goal was speed. A 2 nd order model was necessary for all propagation modes. For the characteristic admittance, a 1 st order model was sufficient for the two line modes, whereas the ground mode required a 3 rd order model. Table 1 shows the TSR coeficients for the propagation and the characteristic admittance modal transfer functions. $\alpha$ and $\beta$ denote the two line modes and 0 denotes the ground mode.

Modal decomposition allows to chose different model orders and propagation delays for each mode. In particular, the time delay used for the $\alpha, \beta$ and 0 modes was 200, 201 and 210 time-steps. respectively. This permitted very low order models with reasonable accuracy.

Figure 3 shows the square-wave response of the ground-mode propagation model and Figure 4 shows the same response of the $\beta$. mode propagation model. Curve 1 is again calculated by FFT pairs while Curve 2 is the TSR approximation. The error in the modaldomain models is of course greater than that in the phase-domain models. but it is still very smail.

Sparsity proved to be very useful in high order models for the ground mode. However, the need of high order models to achieve accuracy for that mode is hardly justified because of the uncertainty in the data involving the ground-return path.

\subsection{Transmission Line Model Validation}

The propagation and characteristic admittance models were validated separately, as shown previously, by frequency and time-domain calculations. The complete transmission line model is validated by comparing periodic waveforms to results obtained by FFT pairs. Our test-system is composed of the transmission line with a source at one end and a load at the other. The source is a square-wave voltage

\begin{tabular}{|c|c|c|c|c|c|c|}
\hline \multirow{2}{*}{ TSR } & \multicolumn{3}{|c|}{$H_{p}$} & \multicolumn{3}{|c|}{$H_{Y}$} \\
\hline & $\alpha$ & $\beta$ & 0 & $\alpha$ & $\beta$ & 0 \\
\hline $\begin{array}{l}A_{0} \\
A_{1} \\
A_{2} \\
A_{3}\end{array}$ & $\begin{array}{r}1.0 \\
-1.977 \\
0.977\end{array}$ & $\begin{array}{r}1.0 \\
-1.957 \\
0.957\end{array}$ & $\begin{array}{r}1.0 \\
-1.959 \\
0.959\end{array}$ & $\begin{array}{c}1.0 \\
-1.000\end{array}$ & $\begin{array}{c}1.0 \\
-1.000\end{array}$ & $\begin{array}{c}1.0 \\
-0.993 \\
-1.0 \\
0.993\end{array}$ \\
\hline $\begin{array}{l}B_{0} \\
B_{1} \\
B_{2} \\
B_{3}\end{array}$ & $\begin{array}{r}0.907 \\
-1.791 \\
0.884\end{array}$ & $\begin{array}{r}0.823 \\
-1.604 \\
0.781\end{array}$ & $\begin{array}{r}-0.192 \\
0.421 \\
-0.229\end{array}$ & $\begin{array}{r}0.002 \\
-0.002\end{array}$ & $\begin{array}{r}0.001 \\
-0.001\end{array}$ & $\begin{array}{r}0.001 \\
-0.001 \\
-0.001 \\
0.001 \\
\end{array}$ \\
\hline
\end{tabular}




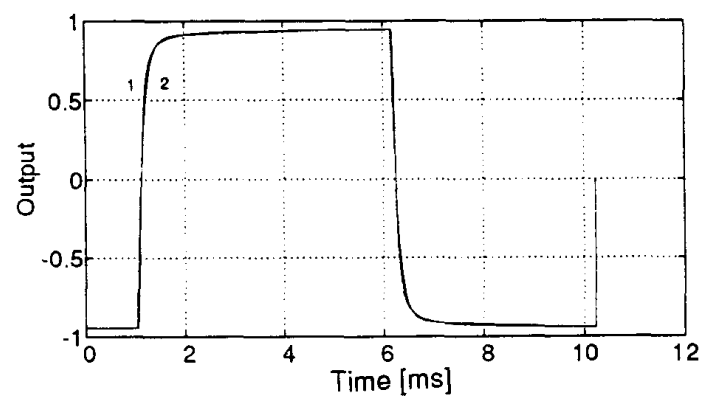

Figure 3. Response to square-wave input of ground-mode propagation model.

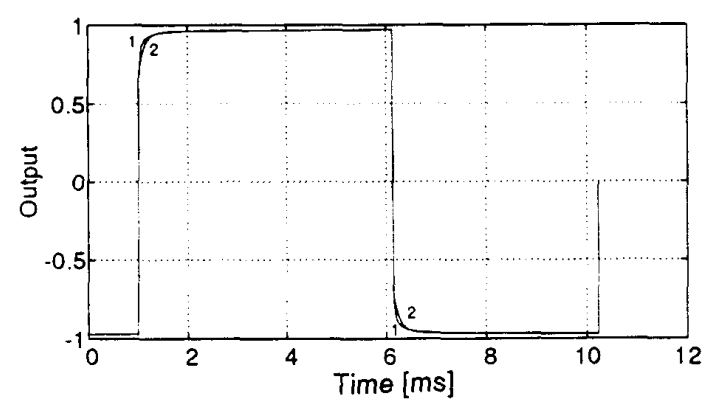

Figure 4. Response to square-wave input of $\beta$-mode propagation model.

source, either general, or exciting a particular mode. For simplicity, because the waveforms are much simpler to present and discuss, we consider the open-circuited case where the period and timing of the square-wave input is such that the negative switching occurs at the instant when the wave returns to the source end. We refer to this situation as the "resonance case".

Figure 5 shows the voitage of phase $a$ at the open-circuited end for ground-mode excitation. that is for three synchronized squarewave inputs, one at each phase. Figure 6 shows the same voltage for $\beta$-mode excitation. that is for two opposite square-wave inputs on phases $a$ and $c$, while phase $b$ is not energized. The period of the input is $20 \mathrm{~ms}$. Curve 1 is obtained by FFT pairs using the $A, B, C$, and $D$ parameters of the line, as calculated in Appendix A. Curve 2 is the TSR approximation using the phase-domain model and Curve 3 is the TSR approximation using the modal-domain models. For the modal-domain models, the frequency of $10 \mathrm{kHz}$ was selected for the calculation of the modal transformation matrices.

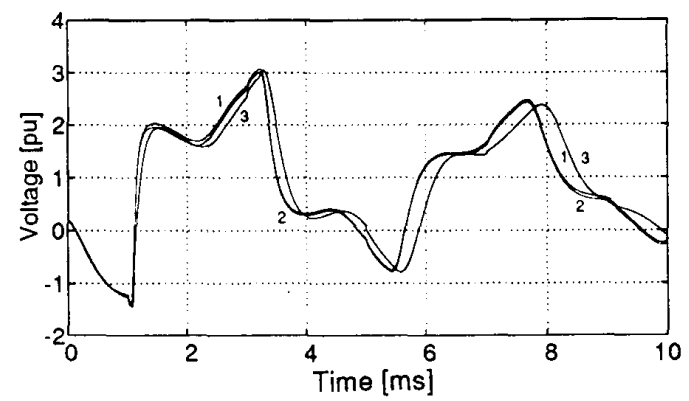

Figure 5. Model validation for ground-mode excitation: open-circuit resonance case.

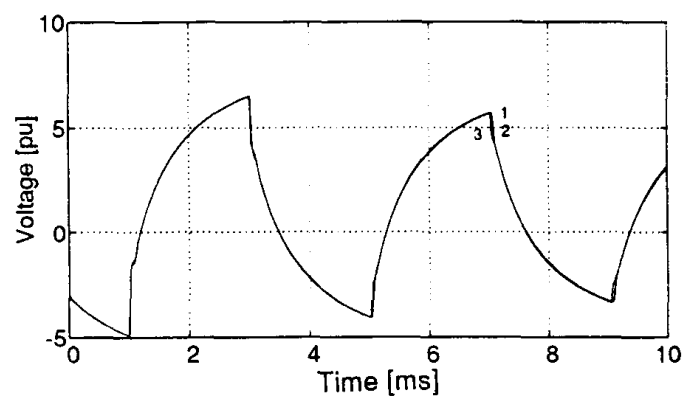

Figure 6. Model validation for $\beta$-mode excitation; open-circuit resonance case.

The high voltage peaks in Figure 5 and Figure 6 are caused by the resonance phenomenon of the voltage source amplifying the traveling waves on the transmission line. We can see that the phasedomain model is very accurate in both cases. The modal-domain models are accurate for the line-mode excitation, but there is a considerable error when the ground mode is excited. The reason is that the frequency dependence of the ground mode is much greater than that of the line modes. This also requires higher-order models for the ground mode. However, as mentioned earlier, a larger error tolerance is acceptable for the ground mode.

\subsection{Transient Simulations}

The transient simulation program is general enough to handle complete networks with several transmission lines and loads. For the purpose of demonstration and discussion. we will consider the simple case of single-line power-frequency simultaneous energization. The line is energized in open-circuit from a three-phase $50 \mathrm{~Hz}$ positive sequence voltage source at the peak voltage of phase $a$.

Figure 7 shows the voltage of phase $a$ at the open end and Figure 8 shows the charging current of the same phase at the source end. Curve 1 is the TSR simulation using the phase-domain model and Curve 2 corresponds to the modal-domain models. Again, the modal-domain models show some difference with respect to the phase-domain model since the simultaneous energization partly excites the ground mode.

\subsection{Traditional Approach Simulations}

For the purpose of comparison, the above simulation was also performed in the traditional way, using rational fitting approximation of the modal transfer matrices and the state variable approach. The relevant calculations and details are contained in Appendix D. Curve 3 in Figure 7 and Figure 8 corresponds to this approach. We can see that the traditional approach is in very good agreement with the new method. In fact, judging from the previous validation

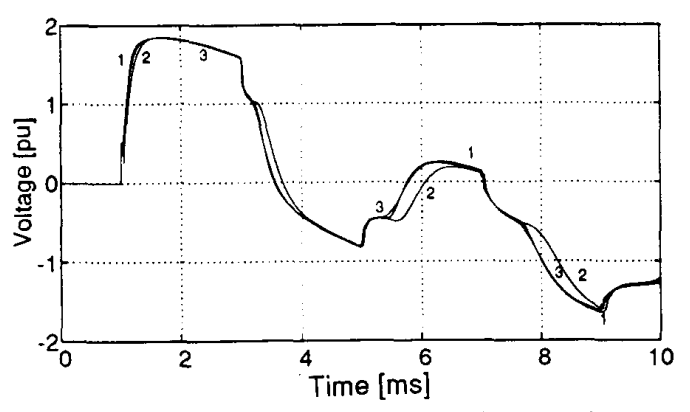

Figure 7. Transient simulation - open-circuit voltage; power frequency open-circuit energization. 


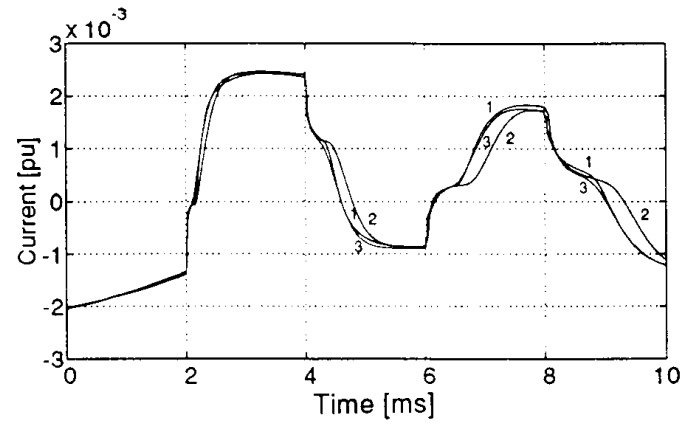

Figure 8. Transient simulation - charging current; power frequency open-circuil energization.

results, taking Curve 1 as the most accurate, we can confirm that the traditional approach is fully satisfactory for the calculation of transients in overhead transmission lines.

The modal TSR method and the traditional approach, for models of the same order, require roughly similar computational effort. The phase-domain TSR method, on the other hand, although it does not involve phase-to-mode back and forth transformations, requires greater computational effort than a low-order traditional approach because of the higher-order matrix-vector models used to achieve the desired accuracy.

\section{CONCLUSIONS}

The methodology developed in this paper for the direct phase-domain calculation of transmission line transients is based on the realization of matrix transfer functions $H(s)$ in the form of two-sided recursions (TSR) with matrix coefficients $A_{k}, B_{k}$, relating inputs to outputs. The TSR coefficients are identified directly from $H(s)$ over a wide range of frequencies without any intermediate procedures, such as rational approximation, that could be very complex in the multi-input/output case.

While the approach described is generally applicable to a multitude of engineering problems and in particular in power system analysis, the application presented here is appropriate to demonstrate both its feasibility and accuracy. In the direct simulation of transients in the phase-domain, the use of TSR gives accurate results even if the modal transformation matrices are frequency dependent. While this is not of serious concem in the case of overhead lines, it could produce great difficulties in the case of cables. This problem is now open for investigation using the TSR approach.

The new method is eminently appropriate for direct phasedomain simulations. However, it could also be used in the modaldomain as an alternative to the highly successful traditional methods based on modal decompositions.

\section{ACKNOWLEDGEMENTS}

The authors gratefully acknowledge financial support by the National Sciences and Engineering Research Council of Canada.

\section{REFERENCES}

11] A. Semlyen and A. Dabuleanu. "Fast and Accurate Switching Transient Calculations on Transmission Lines With Ground Return Using Recursive Convolutions", IEEE Transactions on Power Apparatus and Systems, Vol. PAS-94, No. 2 , pp. 561 -571, March/April 1975.

[2] A. Semlyen, "Contributions to the Theory of Calculation of Electromagnetic Transients on Transmission Lines with Frequency Dependent Parameters", IEEE Transactions on Power Apparatus and Systems, Vol. PAS-100, No. 2, pp. $848-856$, February 1981.

[3] J.R. Marti, "Accurate Modeling of Frequency-Dependent Transmission Lines in Electromagnetic Transient Simulations". IEEE Transactions on Power Apparatus and Systems. Vol. PAS -101. No. 1. pp. 147-157. January 1982.
[4] A. O. Soysal and A. Semlyen, "State Equation Approximation of Transfer Matrices and its Application to the Phase Domain Calculation of Electromagnetic Transients", Paper no. 93 WM $240-2$ PWRS, presented at the IEEE/PES 1993 Winter Meeting in Columbus, Ohio.

[5] L. Marti, "Simulation of Transients in Underground Cables with Frequency-Dependent Modal Transformation Matrices", IEEE Transactions on Power Delivery, Vol. PWRD - 3. No. 3. pp. $1099-1110$. July 1988.

[6] A. Abur and H. Singh, "Time Domain Modeling of External Systems for Electromagnetic Transients Programs", IEEE Transactions on Power Systems, Vol. PWRS-8, No. 2, pp. $671-679$, May 1993.

[7] J. R. Smith. J. F. Hauer, and D. J. Trudnowski, "Transfer Function Identification in Power System Applications". IEEE Transactions on Power Systems, Vol. PWRS -8, No. 3. pp. $1282-1290$, August 1993.

[8] J.F. Hauer, "Power System Identification by Fitting Structured Models to Measured Frequency Response", IEEE Transactions on Power Apparatus and Systems, Vol. PAS - 101. No. 4. pp. 915 -923, April 1982.

[9] W. D. Humpage and K. P. Wong, "Electromagnetic Transient Analysis in EHV Power Networks", IEEE Proceedings, Vol. 70, No. 4, pp. 379-402, April 1982.

[10] G. Dahlquist and A. Björck. Numerical Methods. PrenticeHall, Englewood Cliffs. New Jersey, 1974.

\section{APPENDICES}

\section{Appendix A. Transmission Line Transfer Matrices}

\section{Phase Domain Transfer Matrices}

The voltage and current waves on an overhead transmission line are given by

$$
\begin{gathered}
v=e^{-\sqrt{Z Y} x} v_{o} \\
i=e^{-\sqrt{Y Z x}} i_{o}
\end{gathered}
$$

where $Z$ and $Y$ are the per-unit-length series impedance and shunt admittance matrices, respectively, $x$ the distance along the line and $v_{o}=\left.v\right|_{x=0}$ and $i_{o}=\left.i\right|_{x=0}$. From (A.1) and (A.2), the following wave propagation transfer matrices are defined

$$
\begin{aligned}
& H_{P_{\nu}} \triangleq e^{-\sqrt{Z Y} l} \\
& H_{P_{i}} \triangleq e^{-\sqrt{Y Z} l}
\end{aligned}
$$

where $l$ is the length of the line. Since matrices $Z$ and $Y$ are symmetric, therefore.

$$
H_{P_{\nu}}=H_{P_{i}}^{\mathrm{T}}
$$

The characteristic admittance transfer matrix is given by

$$
H_{Y} \triangleq Z^{-1} \sqrt{Z Y}=(\sqrt{Y Z})^{-1} Y
$$

It can be proved that $H_{Y}$ is symmetric.

\section{Modal Decomposition}

Modal decomposition of the matrix product $Z Y$ and its transpose yields:

$$
\begin{aligned}
& Z Y=T_{v} \wedge T_{v}^{-1} \\
& Y Z=T_{1} \wedge T_{i}^{-1}
\end{aligned}
$$

where $\Lambda$ is the diagonal matrix of eigenvalues, and $T_{v}$ and $T_{i}$ are the matrices of right and left eigenvectors of $Z Y$, respectively, with $T_{\nu}^{\mathrm{T}}=T_{i}^{-1}$. They are also referred to as modal transformation matrices because of the following definition of modal voltage $v_{m}$ and modal current $i_{m}$ :

$$
\begin{gathered}
v \triangleq T_{v} v_{m} \\
i \triangleq T_{i} i_{m}
\end{gathered}
$$

Substituting (A.9) in (A.1) and using (A.7), the wave propagation modal transfer matrix is obtained as follows: 


$$
H_{P_{m}}=T_{\nu}^{-1} H_{P_{v}} T_{v}=T_{i}^{-1} H_{P_{i}} T_{i}=e^{-\sqrt{\Lambda} l}
$$

It is, of course, diagonal. Similarly, the characteristic admittance modal transfer matrix is given by

$$
H_{Y m}=T_{i}^{-1} H_{Y} T_{v}
$$

and is also diagonal.

\section{$A, B, C$, and D Parameters}

Manipulating (A.1) and (A.2) and taking into account both incident and reflected waves on the line, the phase-domain $A, B, C$, and $D$ transfer matrices of the line are obtained as follows

$$
\left[\begin{array}{ll}
A & B \\
C & D
\end{array}\right]=\left[\begin{array}{ll}
\cosh (\sqrt{Z Y} l) & \sinh (\sqrt{Z Y} l) H_{Y}^{-1} \\
\sinh (\sqrt{Y Z} l) H_{Y} & \cosh (\sqrt{Y Z} l)
\end{array}\right]
$$

where, clearly, $A=D^{\mathrm{T}}$.

\section{Appendix B. Stability of TSR Coefficients}

Assuming the recursive relation

$$
y_{k}=\lambda y_{k-1}
$$

(20) can be written as follows:

$$
P(\lambda) y_{0} \equiv\left(A_{n}+A_{n-1} \lambda+A_{n-2} \lambda^{2}+\cdots+A_{0} \lambda^{n}\right) y_{0}=0
$$

With the normalization: $A_{0}=I,(B .2)$ is equivalent to

$$
(M-\lambda I) x=0
$$

where $I$ is the identity matrix, $M$ is the companion matrix of $P(\lambda)$, given by

$$
M=\left[\begin{array}{cccc}
I & & & \\
& I & & \\
& & \ddots & \\
-A_{n}-A_{n-1} & -A_{n-2} & \cdots & -A_{1}
\end{array}\right]
$$

and $x$ consists of the initial values:

$$
x^{\mathrm{T}}=\left[\begin{array}{lllll}
y_{0}^{\mathrm{T}} & y_{1}^{\mathrm{T}} & y_{2}^{\mathrm{T}} & \cdots & y_{n-1}^{\mathrm{T}}
\end{array}\right]
$$

Since (B.3) holds for every eigenpair $(\lambda, x)$ of $M$, the roots of the polynomial equation (B.2) are the eigenvalues of $M$. If all eigenvalues have magnitude less than unity, (B.1) suggests that the coefficients are stable for any set of initial values that constitutes an eigenvector of $M$. Since, any initial value set can be written as a linear combination of the eigenvectors of $M$, the above condition is sufficient for the stability of the model.

\section{Appendix C. Stability Analysis for $\boldsymbol{H}_{\boldsymbol{Y}}$}

The polynomial for the stability analysis of $H_{Y}$ can be obtained from (4) in a similar way as (16) by generalizing the latter for the complex frequency $s$ :

$$
\sum_{k=0}^{n}\left(A_{k} H_{\text {ext }}(s)-B_{k}\right) e^{-k s \Delta t}=0
$$

We have used for its derivation $I e^{s t}$ as input (where again $I$ is the identity matrix) and $H_{e x t}(s) e^{s t}$ as output where $H_{e x t}$ is the external transfer function between the input and output variables. Note that $H_{e x t}$ is not related to $H$.

With the notation

$$
\lambda=e^{s \Delta}
$$

(C.1) can be written in the form

$$
P_{C}(\lambda) \equiv \sum_{k=0}^{n}\left(A_{k} H_{e x t}(s)-B_{k}\right) \lambda^{n-k}=\sum_{k=0}^{n} C_{k} \lambda^{n-k}=0
$$

We note that (C.3) is not a true (matrix) polynomial equation since the coefficients $C_{k}$ of $P_{C}(\lambda)$ are themselves functions of $s$. Nevertheless, (C.3) can be used directly when $H_{e x t}$ is constant. Since $H_{\text {ext }}$ can take on any value, (C.3) can be decomposed into two polynomial equations, $P_{A}(\lambda)=0$ (corresponding to (21)), and a similar one in $B_{k}, P_{B}(\lambda)=0$. In the particular case of zero input, $u(t)=0$, i.e., $H_{e x t}(s) \rightarrow \infty, P_{C}(\lambda)$ of (C.3) reduces to $P_{A}(\lambda)$ of $(21)$, as expected. In fact, although we tested the stability of $P_{B}(\lambda)$ for completeness in all our simulations, this proved to be unnecessary as the stability test for $P_{A}(\lambda)$ was sufficient.

Appendix D. Rational Fitting and State Variable Approach

The scalar transfer function is approximated with a rational fitting of the form:

$$
H(s) \simeq \frac{N(s)}{D(s)}=\frac{N_{0}+N_{1} s+\cdots+N_{n} s^{n}}{D_{0}+D_{1} s+\cdots+D_{n} s^{n}}
$$

Similarly to the identification of TSR coefficients, the polynomial coefficients in (D.1) are obtained by the least squares solution of a system of equations created by writting (D.1) for several frequencies. Subsequently, (D.1) is written as the partial fraction expansion

$$
H(s) \simeq \sum_{i=1}^{n} \frac{r_{i}}{s-p_{i}}
$$

where $p_{i}$ are the roots of $D(s)$ and $r_{i}$ the corresponding residues. Equation (1) can now be written as

$$
\begin{aligned}
& x_{i}(s)=\frac{r_{i}}{s-p_{i}} u(s) \\
& y(s)=\sum_{i=1}^{n} x_{i}(s)+H(\infty) u(s)
\end{aligned}
$$

where $x_{i}(s)$ are state variables in the $s$-domain. (D.3) can be written in the familiar state-space representation given by

$$
\begin{aligned}
& \dot{x}=A x+b u \\
& y=c^{\mathrm{T}} x+d u
\end{aligned}
$$

where $A$ is the diagonal matrix containing the poles $p_{i}, b$ is the vector containing the residues $r_{i}, c$ is a vector of ones and $d=H(\infty)$. Integrating (D.4) using for instance the trapezoidal rule, yields an input/output relation of the form of (5).

\section{BIOGRAPHIES}

George Angelidis (Member, IEEE) was born in Athens, Greece, in 1962. He received the Ptychion degree (B.A.Sc.) from the Aristotle University of Thessaloniki in 1984, and the M.A.Sc. and Ph.D. degrees from the University of Toronto in 1988 and 1992, respectively, all in Electrical Engineering. He is currently working as a Postdoctoral Fellow in the Department of Electrical and Computer Engineering at the University of Toronto. His research interests are in computer applications in large-scale electric power systems.

Adam Semlyen (Fellow, IEEE) was born and educated in Rumania where he obtained a Dipl. Ing. degree and his Ph.D. He started his career with an electric power utility and held academic positions at the Polytechnic Institute of Timisoara, Rumania. In 1969 he joined the University of Toronto where he is a professor in the Department of Electrical and Computer Engineering, emeritus since 1988. His research interests include the steady state and dynamic analysis of power systems, electromagnetic transients, and power system optimization. 


\section{Discussion}

Bjørn Gustavsen (Norwegian Electric Power Research Institute Trondheim, Norway) : I would like to congratulate the authors for a clearly written paper describing an efficient method for phase domain calculation of transients on overhead lines. I have the following comments and questions.

As was pointed out by the authors, the usefulness of phase domain transmission models lies mainly in cases where the frequency dependence of the modal transformation matrix cannot be neglected, e.g. vertical twin circuit overhead lines and in particular underground cables. However, the authors have validated their model for the case of a single circuit overhead line where the high frequency time delays of the individual modes are almost equal. It is not obvious to me that their approach will be equally successful in cases were the time delays are very different. This will now be explained :

It is well known that several difficulties of phase domain calculation of transmission line/cable transients are related to the properties of the propagation matrix, Hp. Each element of $\mathrm{Hp}$ is in the phase domain composed of modal components which in general have different time delays. Obtaining a recursive calculation scheme by fitting the elements of Hp in frequency or time domain therefore becomes very difficult : The elements of $|\mathrm{Hp}(\omega)|$, $\operatorname{Real}\{\mathrm{Hp}(\omega)\}$ and $\operatorname{Imag}\{\mathrm{Hp}(\omega)\}$ may contain numerous oscillations, and the front portion of the elements of $\mathrm{Hp}(\mathrm{t})$ may get a complicated shape. Fig. 1 and Fig. 2 demonstrates this for a system of 3 underground single core coaxial cables (cores and sheaths are numbered 1-6)

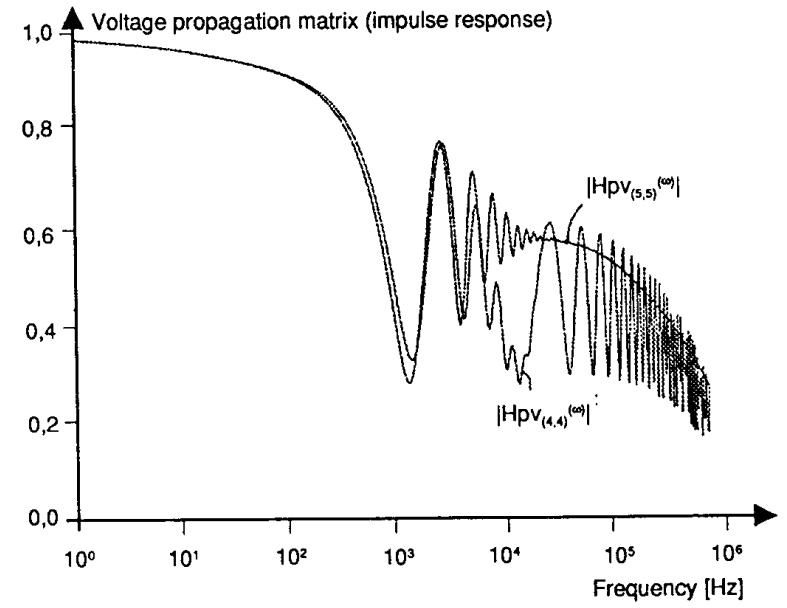

Fig. 1. Elements of the voltage propagation matrix, $|\mathrm{Hpv}(\omega)|$

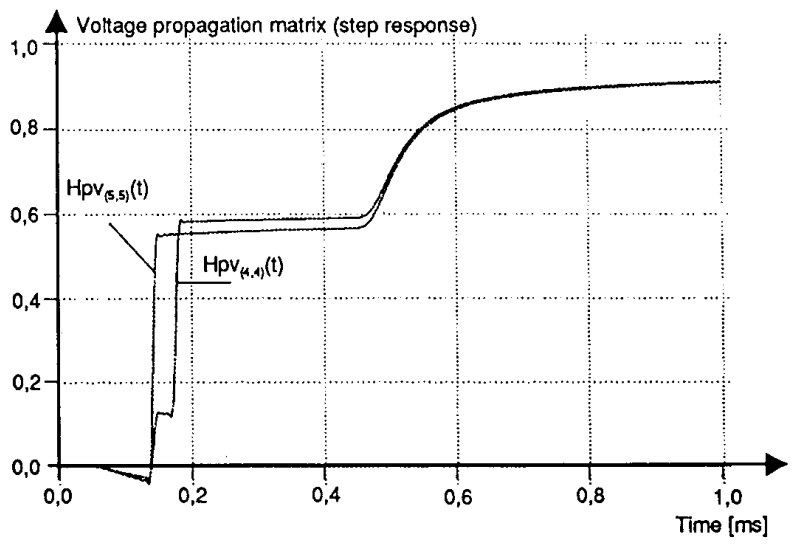

Fig. 2 Corresponding time domain step responses
The identification of TSR coefficients is in the paper based on the real and imaginary parts of $\mathrm{Hp}(\omega)$ (eq. (15)-(18)). In the calculation example (section 3 ), the high frequency time delay of the aerial modes $(\alpha, \beta)$ and the ground mode $(0)$ are almost identical (200, 201 and 210 time steps, respectively). This time delay could therefore easily be removed by multiplying $\mathrm{Hp}(\omega)$ with a single term $\exp (j \omega \tau)$, as was done by the authors, and the frequency domain oscillations of $\operatorname{Real}\{\mathrm{Hp}(\omega)\}$ and $\operatorname{Imag}\{\mathrm{Hp}(\omega)\}$ were consequently removed.

However, it will not be possible to apply this simple procedure to elements of $\mathrm{Hp}$ that are composed of individual modes having very different time delays. This may happen in case of overhead lines (slow ground mode, fast aerial modes), and in particular in some cases of cable transients calculations where all modes need to be taken into account (e.g. crossbonded cables). In these cases, the real and imaginary parts of $H p(\omega)$ will remain oscillatoric after multiplication with the term $\exp (j \omega \tau)$.

I therefore think that the identification of the TSR coefficients may in some cases be considerably more difficult than for the example shown in the paper. Also, I think that the number of TSR coefficients (model order) will in such cases be higher than for the calculation example, due to the complicated shape of the front portion of $\mathrm{Hp}(\mathrm{t})$. I wonder if an increased number of TSR coefficients would make it more difficult to obtain a stable model.

A possible way of circumventing the above problems could be as follows: split the time domain responses of $\mathrm{Hp}$ into a front portion and a tail portion where the tail portion contains the smooth part of the response. Identify the TSR coefficients for the tail portion only (in time domain), and take the front portion into account using ordinary numerical convolution. This splitting technique has been successfully applied in [A].

I would appreciate very much the authors comments on the above discussion.

[A] B. Gustavsen, J. Sletbak and T. Henriksen, "Calculation of electromagnetic transients in transmission cables and lines taking frequency dependent effects accurately into account", paper no. 94 SM 466-3 PWRD, presented at the IEEE/PES 1994 Summer Meeting in San Francisco.

Manuscript received August 18, 1994.

A. AMETANI (Doshisha University, Kyoto 610-03, Japan): The authors are to be congratulated for their valuable contribution to the literature of the transient analysis of transmission lines. The idea of a direct, phase-domain approach was first revealed by this discusser in 1986 /A/. It had the advantage of avoiding convolution of the frequency-dependent transformation matrix $[T]$ as originally proposed by the discusser in $1973 / \mathrm{B} /$. Because the impulse responses of the propagation constant and the characteristic admittance are rather oscillatory in the phase domain, recursive convolution was not applied. But the authors now seem to have successfully applied recursive convolution to the phase-domain components. For this, accuracy of the curve fitting is essential. What method is being used? In Fig. 1, the frequency response is shown only through $100 \mathrm{KHz}$. But for typical transients measured in microseconds, response through several $\mathrm{MHz}$ is required. How accurate is the proposed approximation above $100 \mathrm{KHz}$ ?

The FFT, a discrete Fourier transform, is notorious for its poor accuracy $/ C$, in general, and its need for a large sample size, and substantial memory. The information about the number of samples $\mathrm{N}$, the frequency increment $\Delta \mathrm{f}$, the time step $\Delta \mathrm{t}$, and the maximum observation time Tmax for Figs. 2 through 8 is useful. 
In Figs. 2 through 4, the oscillating periods of the first half and the second half are clearly different. Why? Also, what happens after $10 \mathrm{~ms}$ ? Does the time-response become zero?

The paper shows calculations only for a horizontal overhead line. For this case, the frequency dependence of the transformation matrix $[T]$ is relatively small, however. Far greater variation occurs for an untransposed, vertical, double-circuit line, in which case the variation may even exceed that for cables. Frequency dependence of [T] might reasonably be ignored for the authors' line, but not for all overhead lines (or for most cables, which the authors do not consider).

The authors' observations about the preceding would be most appreciated.

\section{References:}

/A/ H. Nakanishi and A. Ametani, "Transient calculation of a transmission line using superposition law," IEE Proc., Vol. 133C, No. 5, pp. 263-269, 1986.

/B/ A. Ametani, "Refraction coefficient method for switching surge calculations on untransposed transmission lines -- accurate and approximate inclusion of frequency dependence," paper number C73-444-7 presented at the 1973 IEEE PES Summer Meeting.

/C/ A. Ametani and K. Imanishi, "Development of exponential Fourier transform and its application to electrical transients," Proc. IEE, Vol. 126, pp. 51-56, 1979.

Manuscript received August 19, 1994.

George Angelidis and Adam Semlyen (University of Toronto): We would like to thank Dr. Bjorn Gustavsen and Professor Akihiro Ametani for their interest in our paper and for their thoughtful remarks. It is an indication of the fact that direct phase domain calculations are of significance in a number of practical applications.

We agree with the discussers' concern regarding the application of the Two-Sided Recursion methodology for both double-circuit vertical transmission lines and cables. In these cases, the frequency dependence of the transfer functions may present a more difficult problem for fitting. Therefore, as suggested, a higher order TSR may be needed for achieving good accuracy. This, however, does not necessarily create stability problems. In our experience, problems of stability may arise primarily due to spurious modes produced when the order of the TSR is increased to achieve very high accuracy. When a higher order fitting is prompted by the shape of the curve to be approximated, the problem is well-conditioned and instability is not likely to occur. In the following we shall address the individual issues raised by the discussers.

Dr. Gustavsen presents interesting curves in the frequency and time domain. His Figure 1 shows the strongly oscillatory nature of the propagation transfer function $H_{P}$. This is due to the fact that $H_{P}$ has not been smoothed by backwinding (multiplication by $e^{\mathrm{j} \omega \tau}$ ). Otherwise it would appear similar to the upper part of Figure 1 in the paper. The oscillations that would still remain correspond to the additional time delays at the front of the related step response, shown in Figure 2 of the discussion. There is no particular difficulty in fitting the smoothed curve of the transfer function.

Professor Ametani refers to the irregularities in impulse responses and the related difficulty of using recursive convolutions. This is indeed so. We would like at this point to emphasize that TSR is not a convolution (full or recursive) with the input only, but with both input and output. It is equivalent to the discretized form in the course of solving ordinary differential equations (say, with the trapezoidal rule or some multi-step algorithm). Thus, using TSR is a more powerful procedure than that of recursive convolutions, with better capabilities for fitting irregularities in the underlying curves.

Regarding the accuracy of the identification at higher frequencies, we note that in the case of Figure 1 we did not strive to obtain accurate results beyond $100 \mathrm{kHz}$ because the time step was 5 $\mu \mathrm{s}$. We could have gone to higher frequencies for simulations performed with smaller time step.

In order to obtain good accuracy with the FFT for validation purposes, we have used 8192 samples but 4096 is quite satisfactory.

We have noticed, too late, the plotting misprint in Figures 2 to 4. The signals are periodic with period of $10.24 \mathrm{~ms}$ and have halfwave symmetry. The switchover point at $5.12 \mathrm{~ms}$ is not marked. The vertical line segment at $10.24 \mathrm{~ms}$ should not exist. The signal repeats periodically from that point on.

The paper we have presented introduces a new methodology for the computation of transients. We believe that future work will prove its usefulness for solving the type of practical problems raised by the discussers.

Manuscript received October 25, 1994. 